\title{
Dural Venous Sinus
}

National Cancer Institute

\section{Source}

National Cancer Institute. Dural Venous Sinus. NCI Thesaurus. Code C102627.

Venous channels within the dura mater of the brain which receives both blood from blood vessels within the brain as well as cerebrospinal fluid then drains into the internal jugular vein. Unlike other blood vessels, dural venous sinuses lack valves and other vessel associated layers. 